\title{
Drug-Related Hypertension Associated with the Efficacy of Apatinib on Hepatocellular Carcinoma
}

This article was published in the following Dove Press journal:

Cancer Management and Research

\author{
Xuejiao Yang \\ ZhenYu Hou \\ KeYun Zhu \\ Su Zhang \\ XiaoYing Gu \\ ZhiWei Wang \\ $\mathrm{Han} \mathrm{Mu}$ \\ HongYuan Zhou \\ Ping Chen \\ XiaoLin Zhu \\ YunLong Cui \\ Qiang Li \\ HuiKai Li \\ Ti Zhang
}

Department of Hepatobiliary Surgery, Tianjin Medical University Cancer Institute and Hospital, Key Laboratory of Cancer Prevention and Therapy, National Clinical Research Center for Cancer, Tianjin, People's Republic of China
Correspondence: Ti Zhang; HuiKai Li Department of Hepatobiliary Surgery, Tianjin Medical University Cancer Institute and Hospital, Key Laboratory of Cancer Prevention and Therapy, National Clinical Research Center for Cancer, 24 Bin Shui Road, Hexi District, Tianjin 300060, China

$\mathrm{Tel} / \mathrm{Fax}+86-22-2335-9984$

Email zhangti@tjmuch.com; tjchlhk@I26.com
Purpose: We retrospectively evaluated the efficacy and safety of apatinib as a first-line treatment for advanced hepatocellular carcinoma (HCC) and explored whether drug-related hypertension (HTN) could predict its efficacy.

Patients and Methods: This retrospective analysis included patients with advanced HCC who received oral treatment with apatinib. We evaluated the effectiveness by overall survival (OS), progression-free survival (PFS), time to progression (TTP), and disease control rate (DCR), and assessed the safety of the drug based on the occurrence of adverse events. In order to explore whether apatinib-related HTN can be used as a predictor of therapeutic effect, patients were divided into an HTN group and a non-HTN group and adjusted for propensity score-matched (PSM) to reduce mixed deviation. Subgroup analyses of negative prognostic factors for advanced HCC were also performed, including alpha-fetoprotein (AFP), Child-Pugh Score, macrovascular invasion, and extrahepatic metastasis.

Results: A total of 208 patients were analyzed, of which $40.9 \%(n=85)$ developed drugrelated HTN. For all patients, the OS was 13.4 months (95\% CI, 12.2-14.6), the PFS was 5.7 months (95\% CI, 5.1-6.3), and the TTP was 6.9 months (95\% CI, 6.0-7.8). The OS of the HTN group and the non-HTN group was 17.4 months $(\mathrm{m})$ and $12.5 \mathrm{~m}(\mathrm{p}=0.001)$, and the PFS was $7.4 \mathrm{~m}$ and $4.7 \mathrm{~m}(\mathrm{p}=0.000)$, respectively. After PSM, the OS $(\mathrm{p}=0.001)$ and PFS $(p=0.003)$ of the HTN group were still significantly better than the non-HTN group. Subgroup analysis suggested that overall survival was significantly longer in patients with HTN when serum AFP $\leq 400 \mu \mathrm{g} / \mathrm{L}$ or extrahepatic metastases. Moreover, OS in the HTN group increased significantly with or without macrovascular invasion. In addition, through the analysis of two groups of patients with PFS $>6 \mathrm{~m}$ and PFS $\leq 6 \mathrm{~m}$, we know that the patients with drug-related HTN may develop resistance later, so they have longer survival time.

Conclusion: Apatinib demonstrates compelling anti-cancer activity and acceptable safety in advanced HCC. Apatinib-related HTN can potentially predict prolonged survival in patients with advanced HCC.

Keywords: VEGFR-2, HTN, HCC, resistance, liver cancer

\section{Introduction}

Hepatocellular carcinoma (HCC) is classified as the world's seventh-highest ranking cancer for morbidity and its fourth-highest for mortality. In 2018, new incidences of liver cancer exceeded 840,000 and the disease caused more than 780,000 deaths. ${ }^{1}$

About $70 \%$ to $85 \%$ of $\mathrm{HCC}$ is a late or unresectable disease at the time of diagnosis and is not recommended for surgical resection or liver transplantation. ${ }^{2}$ Therefore, it is imperative to develop comprehensive treatments to improve the overall survival rate of advanced HCC. Because of their remarkable effects on other cancers, anti-angiogenic drugs have received much attention in the treatment of 
HCC. From the successful Phase III clinical trial of sorafenib in the treatment of HCC in 2008, the recent firstline treatment of lenvatinib and the second-line replacement therapy of regorafenib and cabozantinib have brought new hope to HCC targeted therapy. ${ }^{3-7}$ Angiogenesis is regulated by a number of growth factors that signal through a variety of tyrosine kinase pathways and bind to specific tyrosine kinase receptors. Vascular endothelial growth factor (VEGF; in particular VEGF-A) and its receptors (VEGFRs; in particular VEGFR-2) play a major pro-angiogenic role. ${ }^{8}$ VEGF inhibitors inhibit vascular growth by inhibiting the binding of VEGF to VEGFR-2 and their biological activities, which achieves anti-tumor effects. ${ }^{9,10}$ Considering the overexpression of VEGFR-2 in HCC and its importance in cancer progression, targeting VEGFR-2 might be a good choice.

Apatinib is a highly selective tyrosine kinase inhibitor of VEGFR-2. ${ }^{11}$ A Phase II clinical study reported at the 2014 American Society of Clinical Oncology (ASCO) Annual Meeting showed that apatinib has potential survival benefits in patients with advanced liver cancer. ${ }^{12}$ Our previous data show that apatinib improves the prognosis of patients with advanced HCC. ${ }^{13}$ However, there are many adverse events, especially hypertension (HTN) and proteinuria during the treatment of apatinib, which can lead to dose reduction or termination of medication in some patients with a good response. Some studies have shown that HTN induced by VEGF inhibitors (VEGFIs) is not a side effect of treatment, but a mechanism-dependent targeted toxicity. The occurrence of HTN may indicate the effectiveness of VEGF inhibition and may serve as a predictor for the beneficial outcome of VEGFI treatment. ${ }^{14-16}$

Therefore, our study was designed to assess the efficacy and safety of apatinib in patients with advanced HCC, and evaluate whether apatinib-related HTN can be used as a predictor of its efficacy.

\section{Patients and Methods}

The inclusion criteria included clinically proven unresectable or metastatic advanced HCC, $\geq 1$ unirradiated measurable lesion as defined by Response Evaluation Criteria In Solid Tumors 1.1 (RECIST 1.1); previous HCC systemic therapy $\leq 1$; $\geq 18$ years old, life expectancy $\geq 12$ weeks, Barcelona Clinical Liver Cancer (BCLC) stage B or C, Eastern Cooperative Oncology Group performance status (ECOG PS) score 0-2, Child-Pugh A or B; better organ function, including liver function (bilirubin $\leq 3 \mathrm{mg} / \mathrm{dl}$, AST and ALT $\leq 5$ times the upper limit of normal value), renal function (serum creatinine $\leq 3.0 \mathrm{mg} / \mathrm{dl}$ or creatine creatinine clearance $\leq 40 \mathrm{~mL} / \mathrm{min}$, urine protein $\leq 1+$, or when urine protein analysis $\geq 2+$, urine protein $<1000 \mathrm{mg} / 24$ hours), hematology (absolute neutrophil count $\geq 1.0^{*} 10^{\wedge} 9 / \mathrm{L}$, hemoglobin $\geq 10 \mathrm{~g} / \mathrm{dL}$, platelet $\geq 50^{*} 10^{\wedge} 9 / \mathrm{L}$ ), and coagulation function (International normalization ratio $\leq 1.5$, partial thromboplastin time $\leq 5$ seconds above ULN).

Exclusion criteria included systemic anti-cancer systemic therapy, localized area therapy or surgery within 28 days prior to entry into the study; ascites that were difficult to control; brain metastases with clinical signs or meningeal carcinogenesis; bleeding of esophageal or gastric varices within 3 months prior to the study; acute hepatitis; presence of progressive central nervous system disease; clinically significant bleeding or thrombotic events within 4 weeks prior to study registration; uncontrollable hypertension or proteinuria $\geq 3+$; Child-Pugh class $\mathrm{C}$.

All patients had an initial dose of $500 \mathrm{mg}$ or $250 \mathrm{mg}$ of oral apatinib daily. When the patient experienced grade $3 / 4$ drug-related adverse events (AEs), the dose was adjusted from either $500 \mathrm{mg}$ per day to $250 \mathrm{mg}$ per day, or from $250 \mathrm{mg}$ per day to $250 \mathrm{mg}$ every other day, or dosage was stopped for a few days until the AEs were been alleviated. Treatment continued until the occurrence of both radiological progression, as defined by RECIST, or the occurrence of either unacceptable adverse events or death.

The article mainly evaluated the effectiveness of overall survival (OS), progression-free survival (PFS), Time to Progression (TTP) and disease control rate (DCR) in patients with apatinib treatment, and assessed the safety according to the occurrence of adverse events. Overall survival is defined as the time from the subject's entry into the trial to death for any reason. Progressionfree survival refers to the length of time a subject enters the trial until the tumor has deteriorated or died. Progression time is the time interval between the subject entering the trial and the appearance of imaging progression. In this study, the PSM method was used to reduce the effect of data bias and confounding variables in order to make a more reasonable comparison between the HTN group and the non-HTN group.

\section{Efficacy and Safety Assessment}

Baseline assessment and tumor screening were performed within 21 days prior to taking apatinib. Baseline and assessments were performed every 8 weeks, including physical examination, vital signs, ECOG PS assessment, electrocardiogram, and clinical and laboratory tests (AFP, 
liver function, and renal function). AEs were classified and ranked according to the National Cancer Institute General Terminology Standard (NCI-CTCAE v 4.0). A computed tomography or magnetic resonance imaging scan was performed every 8 weeks and the tumor response was assessed according to RECIST 1.1.

\section{Drug-Related Hypertension}

In patients without hypertension before treatment, drugrelated hypertension (based on CTCAE version 4.0) is defined as a new diagnosis for patients taking apatinib. In patients with hypertension before treatment, drug-related hypertension is defined as having one or both of the following criteria when taking apatinib: (1) dose increase of previous antihypertensive drugs or/and new antihypertensive drugs (2) systolic blood pressure $\geq 160 \mathrm{mmHg}$ or diastolic blood pressure $\geq 100 \mathrm{mmHg}$ (CTCAE version 3-4 grade or higher). ${ }^{17}$

\section{Statistical Analysis}

All statistical analysis of the data in this study was done with SPSS version 24.0 (IBM Corporation, Armonk, NY, USA). Univariate and multivariate analyses were used in all patients to assess independent factors affecting OS and PFS. Differences between groups were assessed using independent sample $t$-test, chi-square test, Kaplan-Meier survival analysis, and log-rank test. A value of $\mathrm{p}<0.05$ was considered to be statistically significant.

\section{Results}

\section{Patient Characteristics}

From December 2015 to July 2018, a total of 245 advanced HCCs met the inclusion criteria and received apatinib treatment, of which 37 were excluded according to exclusion criteria (Figure S1), and a total of 208 patients were analyzed. The date of the last follow-up was April 5, 2019 , with a median follow-up of 16.8 months. A total of 85 patients $(40.9 \%)$ were diagnosed with drug-related HTN, whereas the rest of the patients (59.1\%) did not develop HTN during apatinib treatment. The patient's baseline and disease characteristics are listed in Table 1.

\section{OS, PFS, and Time to Progression (TTP)}

For all patients, the median OS was 13.4 months (95\% CI: 95\% confidence interval; $12.2-14.6$; Figure S2A), the median PFS was 5.7 months (95\% CI, 5.0-6.3; Figure S2C), and the TTP was 6.9 months $(95 \%$ CI, 6.0-7.7; Figure S2E). In order to remove the influence of confounding factors, we performed propensity score matching (PSM). The remaining 142 patients after PSM had an OS of 13.2 months (95\% CI, 12.0-14.8; Figure S2B), a PFS of 6.2 months (95\% CI, 5.2-7.3; Figure S2D), and a TTP of 7.4 months (95\% CI, 6.0-8.7; Figure S2F). Before PSM, the HTN group was superior to the non-HTN group, including the median OS (HTN vs non-HTN, $17.4 \mathrm{~m}$ vs $12.5 \mathrm{~m}, \mathrm{p}=0.001$; Figure $1 \mathrm{~A}$ ), median PFS (HTN vs non-HTN, $7.4 \mathrm{~m}$ vs $4.7 \mathrm{~m}, \mathrm{p}=0.000$; Figure 1C), and median TTP (HTN vs non-HTN, $8.4 \mathrm{~m}$ vs $5.7 \mathrm{~m}, \mathrm{p}=0.001$; Figure 1E). After PSM, the median OS, PFS, and TTP still suggest that HTN can predict better survival and efficacy (Figure 1B, D, F). The median OS of the two groups was $17.4 \mathrm{~m}$ vs $12.3 \mathrm{~m}$ ( $\mathrm{p}=0.001$; Figure $1 \mathrm{~B})$, median PFS was $7.6 \mathrm{~m}$ vs $4.1 \mathrm{~m}(\mathrm{p}=0.003$; Figure $1 \mathrm{D})$, and TTP was $9.0 \mathrm{~m}$ vs $5.5 \mathrm{~m}$ ( $\mathrm{p}=0.001$; Figure $1 \mathrm{~F})$, respectively.

\section{Objective Response Rate (ORR) and DCR}

For all patients, 2 achieved complete response (CR) (1.0\%), 38 achieved partial response (PR) (18.2\%), and 146 achieved stable disease (SD) (70.2\%), with a total ORR of $19.2 \%$ and a DCR of $89.4 \%$. Before PSM, 21 patients had a partial response (24.7\%) and 1 patient had a complete response $(1.2 \%)$ in the HTN group; In the non-HTN group, 17 patients had a partial response (14.6\%) and 1 patient had a complete response $(0.8 \%)$. The ORR and DCR of the two groups were $25.6 \%$ vs $14.6 \%(\mathrm{p}=0.050)$ and $92.9 \%$ vs $87.0 \%(\mathrm{p}=0.251)$, respectively. There were no significant differences between the two groups. After PSM (1:1), the data showed no significant difference in ORR (HTN vs non-HTN, $26.8 \%$ vs $21.1 \%$ $\mathrm{p}=0.556$ ) and DCR (HTN vs non-HTN, $93.0 \%$ vs $87.3 \%$, $\mathrm{p}=0.399$ ) between the two groups (Table 2). Therefore, although the OS, PFS, and TTP of the HTN group are superior to those of the non-HTN group, the ORR and DCR have not been improved (Table 2). We analyzed that apatinib was effective in both the HTN and non-HTN groups, but the nonHTN group may be more susceptible to drug resistance leading to a reduction in survival time and disease control time.

\section{Univariate and Multivariate Analyses}

Univariate and multivariate analyses of factors affecting OS and PFS in all patients are summarized in Table 3. Univariate Log-rank test analysis of this cohort analysis showed that serum AFP levels, BCLC stage, macrovascular invasion, radiofrequency ablation (RF) therapy, and drug-related HTN were associated with OS. Serum AFP levels, macrovascular invasion, lung metastasis, and other site metastases were 
Table I Patients Baseline and Disease Characteristics (Totals Patients, N=208)

\begin{tabular}{|c|c|c|c|c|c|c|}
\hline \multirow[t]{2}{*}{ Variables } & \multicolumn{3}{|l|}{ All Patients } & \multicolumn{3}{|l|}{ PSM Patients } \\
\hline & HTN (N=85) & Non-HTN $(\mathrm{N}=123)$ & P-value & HTN (N=7I) & Non-HTN (N=7I) & P-value \\
\hline Age & & & 0.316 & & & 1.000 \\
\hline$<60$ years & $46(54.1 \%)$ & $76(61.8 \%)$ & & $40(56.3 \%)$ & $40(56.3 \%)$ & \\
\hline$\geq 60$ years & 39 (45.9\%) & 47 (38.2\%) & & 31 (43.7\%) & 31 (43.7\%) & \\
\hline Sex & & & 0.409 & & & 0.802 \\
\hline Male & 76 (89.4\%) & $104(84.6 \%)$ & & $63(88.7 \%)$ & $6 \mathrm{I}(85.9 \%)$ & \\
\hline Female & $9(10.6 \%)$ & 19 (I5.4\%) & & $8(11.3 \%)$ & $10(14.1 \%)$ & \\
\hline ECOG PS & & & 0.888 & & & 0.502 \\
\hline 0 & 40 (47.1\%) & $60(48.8 \%)$ & & $32(45.1 \%)$ & 37 (52.1\%) & \\
\hline 1 & 45 (52.9\%) & $63(51.2 \%)$ & & 39 (54.9\%) & 34 (47.9\%) & \\
\hline Child-Pugh & & & 0.523 & & & 1.000 \\
\hline A & $65(76.5 \%)$ & 88 (7I.5\%) & & $53(74.6 \%)$ & $53(74.6 \%)$ & \\
\hline B & $20(23.5 \%)$ & $35(28.5 \%)$ & & I 8 (25.4\%) & $18(25.4 \%)$ & \\
\hline BCLC & & & 0.724 & & & 0.833 \\
\hline B & I8 (2I.2\%) & $23(18.7 \%)$ & & 15 (21.1\%) & $13(18.3 \%)$ & \\
\hline C & 67 (78.8\%) & $100(81.3 \%)$ & & $56(78.9 \%)$ & $58(81.7 \%)$ & \\
\hline AFP & & & 0.393 & & & 1.000 \\
\hline$<400 \mu \mathrm{g} / \mathrm{L}$ & 52 (6I.2\%) & 67 (54.5\%) & & $43(60.6 \%)$ & $43(60.6 \%)$ & \\
\hline$\geq 400 \mu \mathrm{g} / \mathrm{L}$ & $33(38.8 \%)$ & $56(45.5 \%)$ & & 28 (39.4\%) & 28 (49.4\%) & \\
\hline Initial dose & & & 0.171 & & & 1.000 \\
\hline $250 \mathrm{mg} / \mathrm{d}$ & 76 (89.4\%) & 117 (95.1\%) & & $66(93.0 \%)$ & 65 (9l.5\%) & \\
\hline $500 \mathrm{mg} / \mathrm{d}$ & $9(10.6 \%)$ & $6(4.9 \%)$ & & $5(7.0 \%)$ & $6(8.5 \%)$ & \\
\hline Pre-HTN & $36(42.4 \%)$ & $15(12.2 \%)$ & 0.000 & $28(39.4 \%)$ & $10(14.1 \%)$ & 0.001 \\
\hline MVI & 37 (43.5\%) & $63(51.2 \%)$ & 0.323 & 35 (49.3\%) & $33(46.5 \%)$ & 0.867 \\
\hline EHS & 46 (54.1\%) & 67 (54.5\%) & 1.000 & $36(50.7 \%)$ & $36(50.7 \%)$ & 1.000 \\
\hline Lung & $6(7.1 \%)$ & $12(9.6 \%)$ & 0.619 & $6(8.5 \%)$ & $5(7.0 \%)$ & 1.000 \\
\hline Bone & $6(7.1 \%)$ & $15(12.2 \%)$ & 0.252 & $5(7.0 \%)$ & $5(7.0 \%)$ & 1.000 \\
\hline Lymph node & 34 (40.0\%) & 46 (37.4\%) & 0.772 & $26(36.6 \%)$ & $23(32.4 \%)$ & 0.724 \\
\hline Other & II (I2.9\%) & 14 (I I.4\%) & 0.829 & 8 (II.3\%) & II (I5.5\%) & 0.623 \\
\hline Hepatitis & & & 0.770 & & & 1.000 \\
\hline None & $15(17.6 \%)$ & 24 (19.5\%) & & II (I5.5\%) & $12(16.9 \%)$ & \\
\hline Hepatitis B & 65 (76.5\%) & 95 (77.2\%) & & 57 (80.3\%) & 56 (78.9\%) & \\
\hline Hepatitis C & $3(3.5 \%)$ & $3(2.4 \%)$ & & $2(2.8 \%)$ & $2(2.8 \%)$ & \\
\hline Hepatitis B + C & $2(2.4 \%)$ & I (0.8\%) & & I (I.4\%) & I (I.4\%) & \\
\hline \multicolumn{7}{|l|}{ Prior Treatment } \\
\hline Surgery & $32(37.6 \%)$ & 40 (32.5\%) & 0.462 & 27 (38.0\%) & $23(32.4 \%)$ & 0.598 \\
\hline TACE or TAE & 67 (78.8\%) & 87 (70.7\%) & 0.230 & $56(78.9 \%)$ & $59(83.1 \%)$ & 0.669 \\
\hline RF & 10 (II.8\%) & $15(12.2 \%)$ & 1.000 & $8(11.3 \%)$ & $6(8.5 \%)$ & 0.779 \\
\hline Radiotherapy & $6(7.1 \%)$ & $2(1.6 \%)$ & 0.045 & $3(4.2 \%)$ & $2(2.8 \%)$ & 1.000 \\
\hline Biotherapy & I (I.2\%) & $3(2.4 \%)$ & 0.646 & I (I.4\%) & $2(2.8 \%)$ & 1.000 \\
\hline Other targeted therapies & $5(5.9 \%)$ & I (0.8\%) & 0.043 & $2(2.8 \%)$ & I (I.4\%) & 1.000 \\
\hline
\end{tabular}

Abbreviations: PSM, propensity score-matched; HTN, hypertension; ECOG PS, Eastern Cooperative Oncology Group performance status score; BCLC, Barcelona Clinic Liver Cancer; AFP, alpha-fetoprotein; Pre-HTN, previous hypertension; MVI, macrovascular invasion; EHS, extrahepatic spread; TACE, transcatheter arterial chemoembolization; TAE, transcatheter arterial embolization; RF, radiofrequency ablation.

associated with PFS ( $\leq \leq 0.05)$. Multivariate Cox regression $\quad 0.334-0.739, p=0.001), R F$ treatment $(\mathrm{HR} 0.400 ; 95 \% \mathrm{Cl}$ analysis showed the following independent factors: for OS, $0.182-0.881, \mathrm{p}=0.023$ ) and drug-related HTN (HR 0.520 ; Child-Pugh Score (HR: hazard ratio; 0.496 ; 95\% CI 95\% CI 0.349-0.775, $\mathrm{p}=0.001$ ) were independent factors; for 


\section{A Survival proportions: Survival of Total OS}

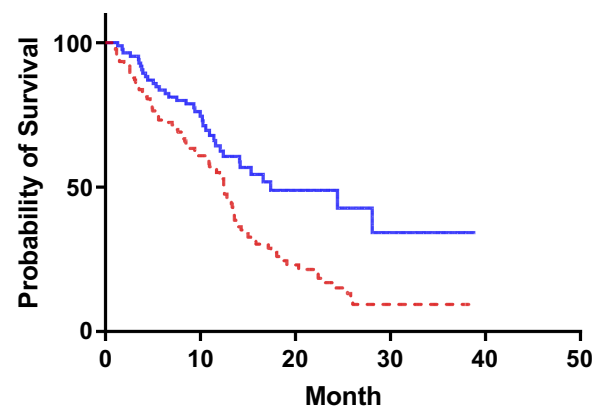

\section{Survival proportions: Survival of Total PFS}

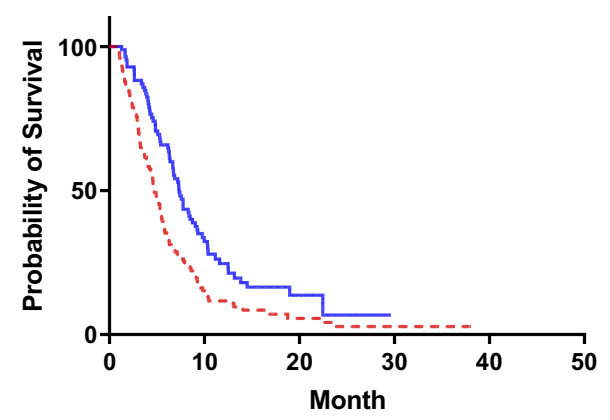

E Survival proportions: Survival of Total TTP

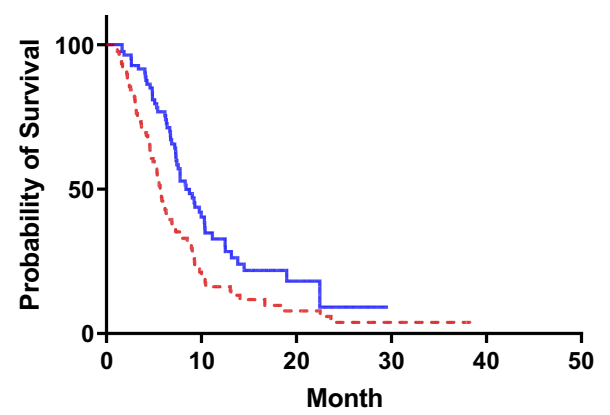

\section{B Survival proportions: Survival of PSM OS}

-.. Non-HTN

- HTN

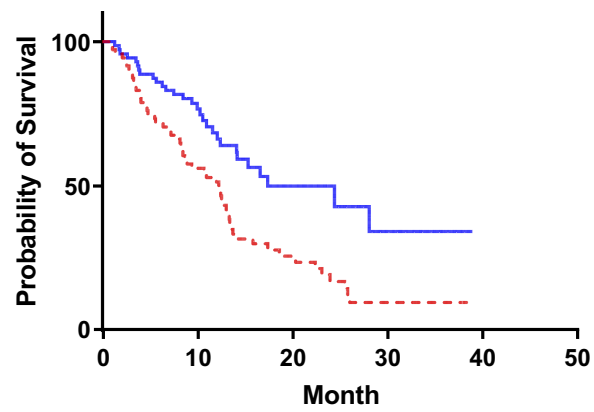

-.. Non-HTN

- HTN
D Survival proportions: Survival of PSM PFS

-.. Non-HTN

- HTN

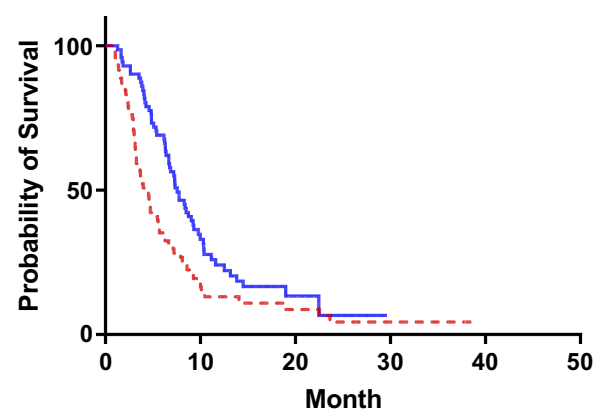

-.. Non-HTN

\section{F Survival proportions: Survival of PSM TTP}

-.. Non-HTN

- HTN

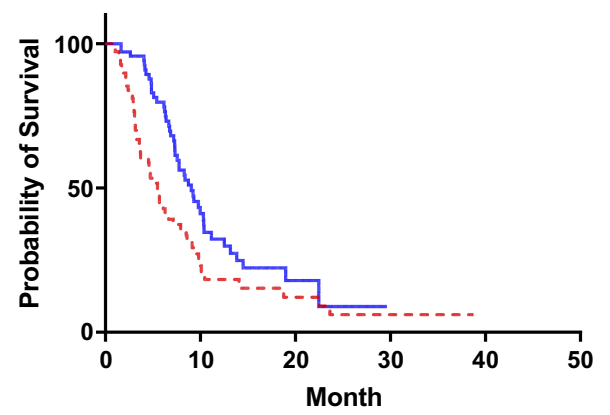

-.. Non-HTN

- HTN

Figure I The overall survival (OS), progression-free survival (PFS), and time to progression (TTP) curves of the HTN and non-HTN groups were plotted using the KaplanMeier method. (A) Overall survival curves of HTN group $(17.4 \mathrm{~m})$ and non-HTN group $(12.5 \mathrm{~m})$ before the PSM (I:I) (Log-rank test, $\mathrm{p}=0.00 \mathrm{I})$. (B) Overall survival curves of HTN group $(17.4 \mathrm{~m})$ and non-HTN group $(12.3 \mathrm{~m})$ after the PSM (I:I) (Log-rank test, $\mathrm{p}=0.001)$. (C) Progression-free survival curves of HTN group $(7.4 \mathrm{~m})$ and non-HTN group $(4.7 \mathrm{~m})$ before the PSM (I:I) (log-rank test, $\mathrm{P}=0.000)$. (D) Progression-free survival curves of HTN group $(7.6 \mathrm{~m})$ and non-HTN group (4.I m) after the PSM (I:I) (Logrank test, $\mathrm{p}=0.003)$. (E) Time to progression curves of HTN group $(8.4 \mathrm{~m})$ and non-HTN group $(5.7 \mathrm{~m})$ before the PSM (I:I) (log-rank test, $\mathrm{p}=0.000)$. $(\mathbf{F})$ Time to progression curves of HTN group $(9.0 \mathrm{~m})$ and non-HTN group $(5.5 \mathrm{~m})$ after the PSM (I:I) (Log-rank test, $\mathrm{p}=0.003)$.

PFS, only drug-related HTN (HR 0.563; 95\% CI 0.413-0.768, $\mathrm{p}=0.001$ ) were independent predictors.

\section{Subgroup Analysis}

Based on the above multivariate and univariate analyses data, we divided patients into HTN and non-HTN groups and analyzed the effects of serum AFP levels, Child-Pugh
Score, macrovascular invasion, and extrahepatic metastases for OS and PFS in the two groups. The results are summarized in Figure 2. In the AFP $\leq 400 \mu \mathrm{g} / \mathrm{L}$ subgroup, the median OS (HTN vs non-HTN, $24.4 \mathrm{~m}$ vs $13.3 \mathrm{~m}, \mathrm{p}=0.006$ ) and PFS (HTN vs non-HTN, $7.8 \mathrm{~m}$ vs $4.7 \mathrm{~m}, \mathrm{p}=0.021$ ) were significantly better in the HTN group than in the non-HTN group; in the $\mathrm{AFP}>400 \mu \mathrm{g} / \mathrm{L}$ subgroup, the presence of drug-related 
Table 2 Short-Term Effects of Patients (Totals Patients, N=208)

\begin{tabular}{|l|l|l|l|l|l|l|l|}
\hline \multirow{2}{*}{ Endpoint } & \multirow{2}{*}{ Totals (N=208) } & \multicolumn{2}{l|}{ All Patients } & \multicolumn{2}{l|}{ PSM Patients } \\
\cline { 3 - 8 } & & HTN (N=85) & Non-HTN (N=I 23) & P-value & HTN (N=7I) & Non-HTN (N=7I) & P-value \\
\hline CR & $2(1.0 \%)$ & $I(1.2 \%)$ & $1(0.8 \%)$ & 1.000 & $I(1.3 \%)$ & 0 & \\
PR & $38(18.2 \%)$ & $21(24.7 \%)$ & $17(13.8 \%)$ & 0.067 & $18(25.4 \%)$ & $15(21.1 \%)$ & 1.000 \\
SD & $146(70.2 \%)$ & $57(67.1 \%)$ & $89(72.4 \%)$ & 0.443 & $47(66.2 \%)$ & $47(66.2 \%)$ & 0.691 \\
ORR (\%) & $40(19.2 \%)$ & $22(25.6 \%)$ & $18(14.6 \%)$ & 0.050 & $19(26.8 \%)$ & $15(21.1 \%)$ & 1.000 \\
DCR (\%) & $186(89.4 \%)$ & $79(92.9 \%)$ & $107(87.0 \%)$ & $0.25 I$ & $66(93.0 \%)$ & $62(87.3 \%)$ & 0.556 \\
\hline
\end{tabular}

Abbreviations: PSM, propensity score-matched; HTN, hypertension; CR, complete response; PR, partial response; SD, stable disease; ORR, objective response rate; DCR, disease control rate.

HTN increased PFS (HTN vs non-HTN, $6.3 \mathrm{~m}$ vs $4.7 \mathrm{~m}$, $\mathrm{p}=0.007$ ) but not OS (HTN vs non-HTN, $15.3 \mathrm{~m}$ vs $12.5 \mathrm{~m}$, $\mathrm{p}=0.081$ ) (Figure 2A and B). In the Child-Pugh A subgroup, HTN can be used as a predictor of PFS (HTN vs non-HTN, $7.6 \mathrm{~m}$ and $5.3 \mathrm{~m}, \mathrm{p}=0.012$ ) growth, but has no significant effect on OS (HTN vs non-HTN, $12.4 \mathrm{~m}$ and $13.5 \mathrm{~m}$, $\mathrm{p}=0.565)$. Similarly, in the Child-Pugh B subgroup, HTN can only predict PFS prolongation (PFS, HTN vs nonHTN, $7.0 \mathrm{~m}$ vs $4.3 \mathrm{~m}, \mathrm{p}=0.010$; OS, HTN vs non-HTN, $9.2 \mathrm{~m}$ vs $9.5 \mathrm{~m}, \mathrm{p}=0.324$ ) (Figure $2 \mathrm{C}$ and $\mathrm{D}$ ). In the subgroup analysis of macrovascular invasion, the median OS (HTN vs non-HTN, $15.3 \mathrm{~m}$ vs $11.0 \mathrm{~m}, \mathrm{p}=0.021$ ) and PFS (HTN vs nonHTN, $7.3 \mathrm{~m}$ vs $4.3 \mathrm{~m}, \mathrm{p}=0.001$ ) were better in the HTN group than in the non-HTN group; in the subgroup of nonmacrovascular invasion, although HTN predicted OS (HTN vs non-HTN, $28.1 \mathrm{~m}$ vs $14.0 \mathrm{~m}, \mathrm{p}=0.024)$ elevation, PFS (HTN vs non-HTN, $7.5 \mathrm{~m}$ vs $5.5 \mathrm{~m}, \mathrm{p}=0.072$ ) could not be expected (Figure $2 \mathrm{E}$ and $\mathrm{F}$ ). In the subgroup analysis of extrahepatic metastases, HTN was a predictor of better OS (HTN vs non-HTN, $24.4 \mathrm{~m}$ vs $12.5 \mathrm{~m}, \mathrm{p}=0.005$ ) and PFS (HTN vs non-HTN, $7.3 \mathrm{~m}$ vs $4.5 \mathrm{~m}, \mathrm{p}=0.000$ ); in subgroups without extrahepatic metastases, HTN predicts a good PFS (HTN vs non-HTN, $7.4 \mathrm{~m}$ vs $5.3 \mathrm{~m}, \mathrm{p}=0.035$ ) but does not mean an increase in OS (HTN vs non-HTN, $15.3 \mathrm{~m}$ vs $12.5 \mathrm{~m}$, $\mathrm{p}=0.078$ ) (Figure $2 \mathrm{G}$ and $\mathrm{H}$ ). In each of the above subgroups, only non-microvascular infiltration was not a predictor of PFS improvement in the HTN group.

We divided all patients into PFS $\leq 6.0 \mathrm{~m}$ and PFS $>6.0 \mathrm{~m}$ for OS comparison (Figure 3). The results showed that OS (PFS $\leq 6.0 \mathrm{~m}$ vs $P F S>6.0 \mathrm{~m}, 8.6 \mathrm{~m}$ vs $20.3 \mathrm{~m}, \mathrm{p}=0.000$ ) significantly prolonged when PFS $>6.0 \mathrm{~m}$. Moreover, patients with drug-related HTN were more likely to achieve PFS $>6.0 \mathrm{~m}$, and another group had more early progression (PFS $\leq 6.0 \mathrm{~m}$, HTN vs non-HTN, $35.0 \%$ vs $68.9 \%$, $\mathrm{p}=0.000$; PFS $>6.0 \mathrm{~m}$, HTN vs non-HTN, $65.0 \%$ vs $34.1 \%$, $\mathrm{p}=0.000$ ). Surprisingly, HTN did not cause significant differences when comparing the survival effects of HTN in patients with PFS $\leq 6.0 \mathrm{~m}$ and PFS $>6.0 \mathrm{~m}$, respectively (PFS $\leq 6.0 \mathrm{~m}$, HTN vs non-HTN, $7.5 \mathrm{~m}$ vs $8.6 \mathrm{~m}, \mathrm{p}=0.469$; PFS $>6.0 \mathrm{~m}, \mathrm{HTN}$ vs non-HTN, $28.1 \mathrm{~m}$ vs $18.3 \mathrm{~m}, \mathrm{p}=0.196$ ). In summary, patients with drug-related HTN may develop drug resistance later, thus prolonging survival.

\section{Safety}

The adverse events of apatinib treatment are listed in Table 4. In this study, $90.9 \%$ of patients had any grade of AEs, and most of the adverse events were mild to moderate. Grade 3 or 4 adverse events occurred in $38.0 \%$ of patients. The most common AEs were HTN (40.9\%), hand-foot syndrome (39.4\%), fatigue (38.0\%), abnormal liver function (31.7\%), blood toxicity $(27.4 \%)$, anorexia $(26.9 \%)$, proteinuria $(24.0 \%)$ and diarrhea (22.6\%). Thrombocytopenia is the most common hematological toxicity. Level 3 or 4 AEs mainly included proteinuria $(12.0 \%)$, hematologic toxicity (10.6\%), and liver dysfunction (6.7\%). Hepatic dysfunction was assessed by using ALT, AST, and other indicators. No grade 5 toxicity occurred in all patients. All AEs can be controlled by dose reduction or withdrawal.

\section{Discussion}

In the present study, we retrospectively confirmed the efficacy and safety of apatinib as a first-line treatment on 208 advanced HCC patients and explored drug-related HTN as a predictor of efficacy. The study showed that the efficacy of apatinib in the treatment of advanced unresectable or metastatic HCC was 13.4 months for OS, 5.7 months for PFS. The rates of CR, PR, SD and DCR were $1.0 \%, 18.2 \%, 70.2 \%$ and $89.4 \%$, respectively.

Based on previous studies and literature reports, patients with drug-related HTN had better efficacy. ${ }^{18,19}$ This study confirmed this hypothesis. In the current study, patients with drug-related HTN had a significantly 
Table 3 Univariate and Multivariate Analyses of Variables Affecting OS and PFS in Patients (Totals Patients, N=208)

\begin{tabular}{|c|c|c|c|c|c|c|c|c|}
\hline \multirow{3}{*}{ Variable } & \multicolumn{4}{|l|}{ os } & \multicolumn{4}{|l|}{ PFS } \\
\hline & \multicolumn{2}{|l|}{ Univariate } & \multicolumn{2}{|l|}{ Multivariate } & \multicolumn{2}{|l|}{ Univariate } & \multicolumn{2}{|l|}{ Multivariate } \\
\hline & HR $(95 \% \mathrm{Cl})$ & $P$ value & HR $(95 \% \mathrm{Cl})$ & $P$ value & HR $(95 \% \mathrm{Cl})$ & $P$ value & HR $(95 \% \mathrm{Cl})$ & $P$ value \\
\hline HTN & $0.28 I(0.156-0.503)$ & 0.000 & $0.520(0.349-0.775)$ & 0.001 & $0.340(0.143-0.812)$ & 0.015 & $0.563(0.4 \mid 3-0.768)$ & 0.001 \\
\hline Pre-HTN & $1.825(0.964-3.454)$ & 0.065 & & & $1.532(0.618-3.796)$ & 0.357 & & \\
\hline $\begin{array}{l}\text { Sex (male vs } \\
\text { female) }\end{array}$ & $0.560(0.234-1.339)$ & 0.192 & $1.035(0.629-1.702)$ & 0.892 & $0.000(0.000-)$ & 0.998 & & \\
\hline Age $(<60$ years & I.47। (0.838-2.583) & 0.179 & $1.262(0.852-1.870)$ & 0.246 & $1.131(0.487-2.628)$ & 0.774 & & \\
\hline vs $\geq 60$ ) & & & & & & & & \\
\hline Initial dose & & 0.994 & & & & 0.871 & & \\
\hline $250 \mathrm{mg} / \mathrm{d}$ & $1.004(0.344-2.935)$ & & & & $1.137(0.241-5.363)$ & & & \\
\hline $500 \mathrm{mg} / \mathrm{d}$ & $0.996(0.34 I-2.910)$ & & & & $0.879(0.186-4.148)$ & & & \\
\hline ECOG PS & & 0.756 & & & & 0.664 & & \\
\hline 0 & $0.916(0.525-1.596)$ & & & & $1.205(0.520-2.795)$ & & & \\
\hline I & $1.092(0.627-1.903)$ & & & & $0.830(0.358-1.925)$ & & & \\
\hline AFP & & 0.032 & & 0.620 & & 0.019 & & 0.089 \\
\hline AFP $>400 \mu g / L$ & $1.872(1.054-3.325)$ & & $1.102(0.75 \mid-1.615)$ & & $3.394(1.221-9.433)$ & & $1.301(0.961-1.760)$ & \\
\hline AFP $\leq 400 \mu g / L$ & $0.534(0.301-0.949)$ & & $0.908(0.619-1.331)$ & & $0.295(0.106-0.819)$ & & $0.769(0.568-1.041)$ & \\
\hline Child-Pugh & & 0.000 & & 0.001 & & 0.439 & & 0.169 \\
\hline A & $0.177(0.078-0.399)$ & & $0.496(0.334-0.739)$ & & $0.665(0.237-1.867)$ & & $0.785(0.556-1.108)$ & \\
\hline B & 5.649 & & $2.014(1.353-2.998)$ & & $1.504(0.536-4.223)$ & & $1.274(0.902-1.797)$ & \\
\hline & $(2.503-12.748)$ & & & & & & & \\
\hline BCLC & & 0.197 & & 0.182 & & 0.009 & & 0.322 \\
\hline B & $0.636(0.320-1.265)$ & & $0.602(0.286-1.269)$ & & $0.306(0.126-0.744)$ & & $0.737(0.403-1.349)$ & \\
\hline C & $1.572(0.790-3.127)$ & & $1.661(0.788-3.500)$ & & $3.269(1.344-7.948)$ & & $1.357(0.742-2.483)$ & \\
\hline Hepatitis (-) & & & & & & & & \\
\hline None & $0.944(0.465-1.918)$ & 0.874 & & & $1.835(0.708-4.758)$ & 0.212 & & \\
\hline Hepatitis B & $1.130(0.579-2.209)$ & 0.72 & & & $1.857(0.744-4.634)$ & 0.185 & & \\
\hline Hepatitis C & $0.832(0.214-3.159)$ & 0.776 & & & $1.097(0.1319 .160)$ & 0.932 & & \\
\hline MVI & $1.909(1.081-3.345)$ & 0.026 & $1.037(0.624-1.725)$ & 0.888 & $2.657(1.059-6.669)$ & 0.037 & $1.078(0.718-1.619)$ & 0.716 \\
\hline EHS & 1.007 (0.577-1.759) & 0.979 & $1.312(0.809-2.127)$ & 0.270 & $1.603(0.691-3.719)$ & 0.272 & $0.961(0.639-1.445)$ & 0.848 \\
\hline Bone & $0.873(0.35 I-2.175)$ & 0.771 & & & $2.945(0.378-2.957)$ & 0.303 & & \\
\hline Lung & $1.811(0.620-5.285)$ & 0.277 & & & $1.803(1.106-2.940)$ & 0.018 & & \\
\hline Lymph node & $0.839(0.475-1.482)$ & 0.545 & & & $0.929(0.396-2.182)$ & 0.866 & & \\
\hline Other & $2.971(1.068-8.264)$ & 0.037 & & & $1.961(1.281-3.002)$ & 0.002 & & \\
\hline Prior Treatment & & & & & & & & \\
\hline Surgery & $0.483(0.270-0.866)$ & 0.015 & $0.756(0.500-1.144)$ & 0.185 & $1.416(0.562-3.569)$ & 0.46 & & \\
\hline $\mathrm{RF}$ & $0.265(0.109-0.648)$ & 0.004 & $0.400(0.182-0.881)$ & 0.023 & $0.367(0.131-1.031)$ & 0.057 & $0.768(0.457-1.290)$ & 0.318 \\
\hline TACE or TAE & $1.048(0.557-1.97 I)$ & 0.884 & & & $0.888(0.335-2.355)$ & 0.812 & & \\
\hline Biotherapy & $0.659(0.091-4.769)$ & 0.679 & & & $1.649(0.610-4.462)$ & 0.324 & & \\
\hline Radiotherapy & I.III (0.258-4.780) & 0.887 & & & $0.955(0.113-8.099)$ & 0.966 & & \\
\hline $\begin{array}{l}\text { Other targeted } \\
\text { therapies }\end{array}$ & $0.321(0.057-1.795)$ & 0.196 & $0.429(0.090-2.045)$ & 0.288 & $0.257(0.045-1.482)$ & 0.129 & $0.785(0.261-2.366)$ & 0.668 \\
\hline
\end{tabular}

Abbreviations: OS, overall survival; PFS, progression-free survival; PSM, propensity score-matched; HTN, hypertension; Pre-HTN, previous hypertension; HR, hazard ratio; 95\% Cl, 95\% confidence interval; ECOG PS, Eastern Cooperative Oncology Group performance status score; BCLC, Barcelona Clinic Liver Cancer; AFP, alphafetoprotein; MVI, macrovascular invasion; EHS, extrahepatic spread; TACE, transcatheter arterial chemoembolization; TAE, transcatheter arterial embolization; RF, radiofrequency ablation.

longer median overall survival than those without HTN. Correspondingly, a longer PFS was also observed in the drug-related HTN group. Moreover, the study of Hamnvik OP confirms that the blood pressure increased equally in those with and without pre-existing hypertension. ${ }^{17}$ Therefore, patients with higher baseline blood pressure are more likely to develop drug-related hypertension during treatment. 


\section{A Survival proportions: Survival of AFP OS}

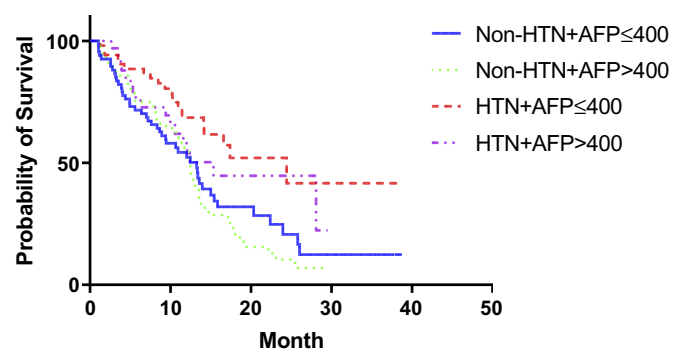

C Survival proportions: Survival of Vascularinvasion OS

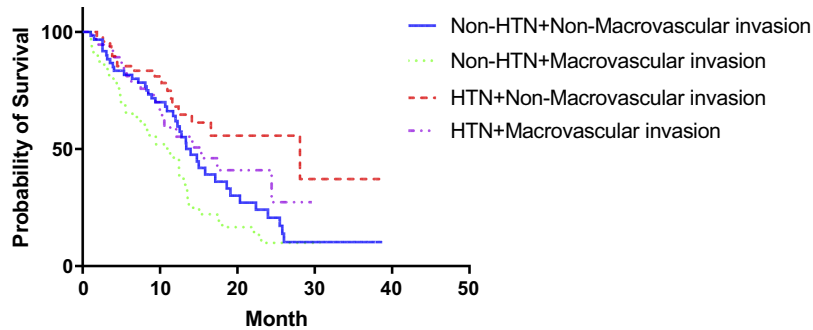

E Survival proportions: Survival of Extrahepatic spread OS

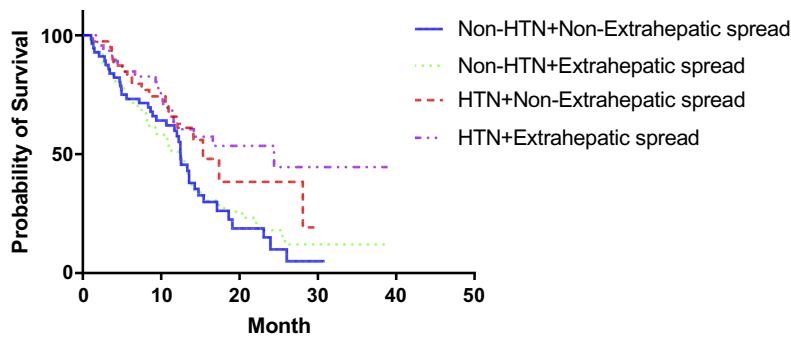

G Survival proportions: Survival of ChildPugh OS

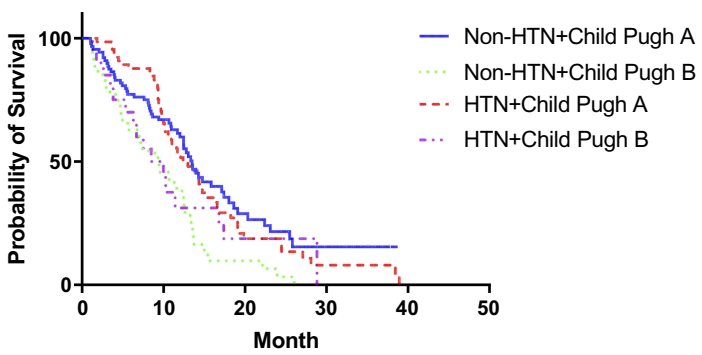

B Survival proportions: Survival of AFP PFS

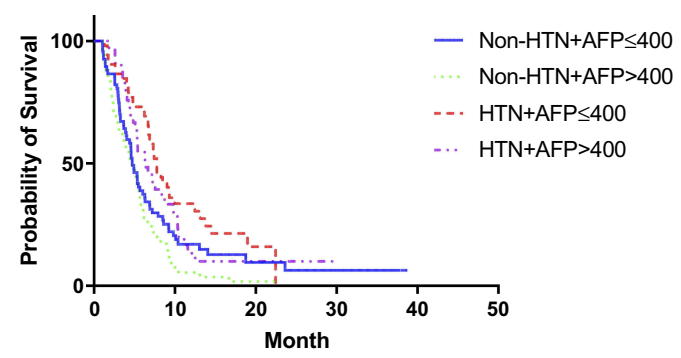

D Survival proportions: Survival of Vascularinvasion PFS

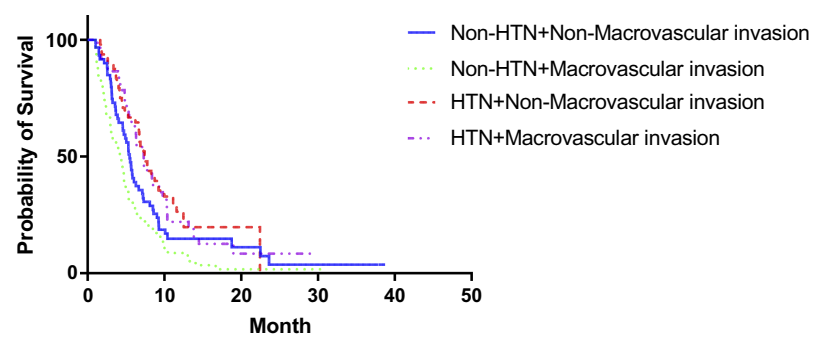

F Survival proportions: Survival of Extrahepatic spread PFS

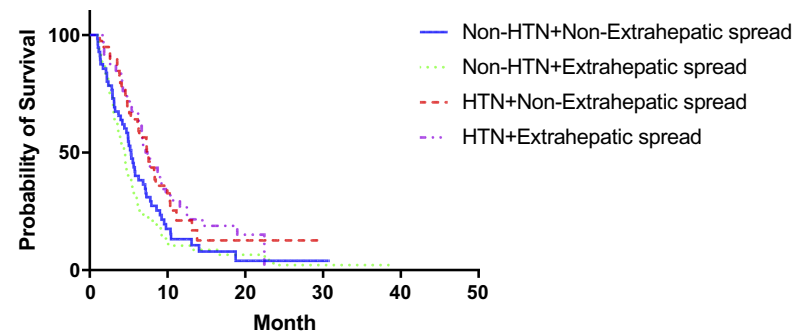

H Survival proportions: Survival of ChildPugh PFS

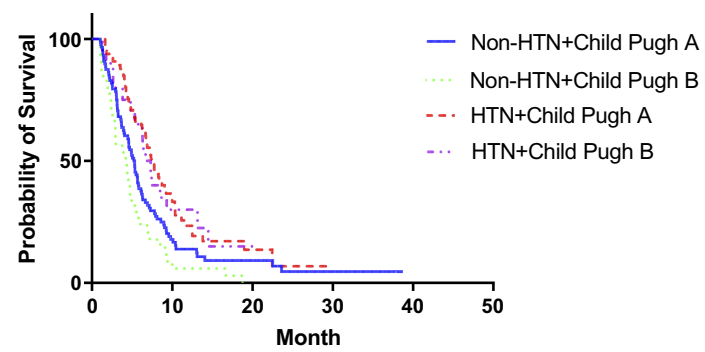

Figure 2 Subgroup analysis of OS and PFS. (A) Subgroup with AFP $>400 \mu g / L$, whether HTN can be a predictor of OS; (B) subgroup with AFP $>400 \mu g / L$, whether HTN can be a predictor of PFS; $(A, B)$ In patients with AFP $\leq 400 \mu \mathrm{g}$, drug-related HTN can be used as a predictor of OS $(p=0.006)$ and PFS ( $p=0.02 \mathrm{I})$. In patients with AFP $>400 \mu \mathrm{g}$, drug-related HTN cannot be used as a predictor of OS $(p=0.08 \mathrm{I})$, but can be used as a predictor of PFS ( $=0.007$ ). (C) Subgroup with Child-Pugh Score, whether HTN can be a predictor of OS; (D) subgroup with Child-Pugh Score, whether HTN can be a predictor of PFS; (C, D) in patients with Child-Pugh A, drug-related HTN can be used as a predictor of OS $(p=0.003)$ and PFS $(p=0.012)$. In patients with Child-Pugh $B$, drug-related HTN cannot be used as a predictor of OS ( $=0.267)$, but can be used as a predictor of PFS $(P=0.010)$. (E) Subgroup with macrovascular invasion, whether HTN can be a predictor of OS; (F) subgroup with macrovascular invasion, whether HTN can be a predictor of PFS; (E, F) in patients without macrovascular invasion, drug-related HTN can be used as a predictor of OS ( $\mathrm{P}=0.024)$, but not as a predictor of PFS $(p=0.072)$. In patients with macrovascular invasion, drug-related HTN can be used as a predictor of OS $(p=0.02 \mathrm{I})$ and PFS ( $p=0.00 \mathrm{I})$. (G) Subgroup with extrahepatic spread, whether HTN can be a predictor of OS; $(\mathbf{H})$ subgroup with extrahepatic spread, whether HTN can be a predictor of PFS. (G, H) In patients without extrahepatic metastases, drug-related HTN cannot be used as a predictor of OS $(p=0.078)$, but can be used as a predictor of PFS ( $p=0.035)$. In patients with extrahepatic spread, drugrelated HTN can be used as a predictor of OS $(p=0.005)$ and PFS $(p=0.000)$. 


\section{Survival proportions: Survival of PFS>6month OS}

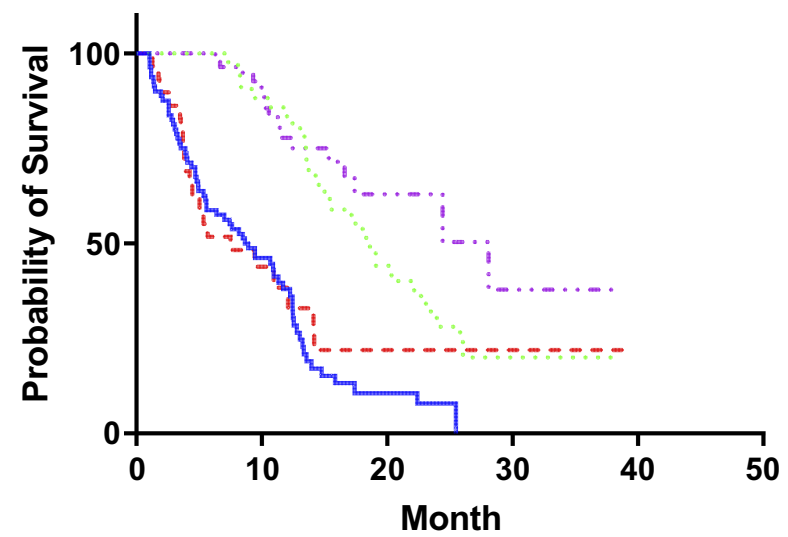

- Non-HTN+PFS $\leq 6$ month Non-HTN+PFS $>6$ month

-.. HTN+PFS $\leq 6$ month

... HTN+PFS $>6$ month

\begin{tabular}{|l|c|c|}
\hline & Non-HTN & HTN \\
\hline PFS $\leq 6 \mathrm{~m}$ & 80 & 29 \\
& & \\
\hline PFS $>6 \mathrm{~m}$ & 43 & 56 \\
\hline \multicolumn{3}{|c|}{$\mathrm{P}=0.000$} \\
\hline
\end{tabular}

Figure 3 The Kaplan-Meier plot shows the overall survival rate for a combination of radiological outcomes based on changes in blood pressure after administration and 6 months later by modified Response Evaluation Criteria in Solid Tumors. The overall survival of patients with PFS $>6$ months was significantly better than patients with PFS $\leq 6$ months, with or without drug-related HTN. However, the drug-related HTN cannot be used as a predictor of OS in these two groups of patients.

In addition, we also observed that patients treated with apatinib had longer TTP than PFS (TTP vs PFS: $6.9 \mathrm{~m}$ vs $5.7 \mathrm{~m}$ before PSM; $7.4 \mathrm{~m}$ vs $6.2 \mathrm{~m}$ after PSM). This is reported to be related to the differing clinical significance of TTP and PFS. In general, PFS analysis included tumor progression time and early death time due to liver failure or other non-tumor causes, but TTP only analyzed the former. In other words, TTP eliminates the death of patients with early treatment failure, resulting in better results.

Table 4 Adverse Events Profile Based on Apatinib Treatment (Totals Patients, $\mathrm{N}=208$ )

\begin{tabular}{|l|l|l|}
\hline Adverse Events & $\begin{array}{l}\text { Any Grade, No. } \\
\text { (\%) }\end{array}$ & $\begin{array}{l}\text { Grade 3 or 4, No. } \\
\text { (\%) }\end{array}$ \\
\hline $\begin{array}{l}\text { All adverse events } \\
\text { Hypertension }\end{array}$ & $\begin{array}{l}189(90.9 \%) \\
85(40.9 \%)\end{array}$ & $\begin{array}{l}79(38.0 \%) \\
1(0.5 \%)\end{array}$ \\
Hand and foot & $82(39.4 \%)$ & $10(4.8 \%)$ \\
syndrome & & $7(3.4 \%)$ \\
Weak & $79(38.0 \%)$ & $14(6.7 \%)$ \\
Abnormal liver & $66(31.7 \%)$ & \\
function & $57(27.4 \%)$ & $22(10.6 \%)$ \\
Hematological & & $12(5.8 \%)$ \\
toxicity & $56(26.9 \%)$ & $25(12.0 \%)$ \\
Anorexia & $50(24.0 \%)$ & $2(1.0 \%)$ \\
Proteinuria & $47(22.6 \%)$ & $6(2.9 \%)$ \\
Diarrhea & $32(15.4 \%)$ & $2(1.0 \%)$ \\
Vomiting & $30(14.4 \%)$ & 0 \\
Hoarse voice & $27(13.0 \%)$ & $7(3.4 \%)$ \\
Dry mouth & $20(9.6 \%)$ & 0 \\
Ascites & $11(5.3 \%)$ & \\
Stomach ache & &
\end{tabular}

Our multivariate analysis showed that Child-Pugh score, RF therapy, and drug-related HTN were independent factors for the median overall survival, while serum AFP level and hypertension were independent predictors of median PFS in patients with apatinib treatment. In the subgroup analysis of patients with drug-related HTN, AFP $\leq 400 \mu \mathrm{g} / \mathrm{L}$ and extrahepatic metastases were predictors of good OS. Moreover, regardless of whether the patient has microvascular invasion, drug-related HTN can be used as a predictor of OS prolongation.

All patients were divided into PFS $>6$ months and PFS $\leq 6$ months. In both groups, drug-related HTN did not predict better OS. However, drug-related HTN is more likely to cause PFS $>6$ months. This suggests that HTN may increase survival time by prolonging the time of drug resistance, but does not continue to affect drug efficacy after disease progression. The most common adverse events for the treatment of advanced HCC with apatinib are consistent with previous studies. HTN, hand-foot syndrome, and fatigue are the most common adverse events. Proteinuria, abnormal liver function, and hematologic toxicity (including thrombocytopenia, leukopenia, etc.) are the most common grade 3-4 adverse events. All adverse events can be alleviated or controlled by symptomatic treatment or dose reduction and withdrawals. Our study has shown that drugrelated HTN can be used as a predictor of the efficacy of apatinib. At present, many studies have confirmed that HTN is a predictive marker for the efficacy of antiangiogenic therapy. ${ }^{20,21}$ Moreover, some articles have shown that the use of appropriate anti-HTN drugs has 
no effect on the clinical outcome of patients. ${ }^{22-24}$ This means that severe HTN should be controlled by taking aggressive medication before reducing the dose or suspending the treatment.

Our research has limitations. First, this study is a retrospective study. It is impossible to weaken the influence of confounding variables between the HTN group and the non-HTN based on the law of large number, and it is easy to produce systematic bias. PSM is used to solve this problem and eliminate interference factors between groups. Secondly, the study is a single-arm, single-center study. Patients are confined to the same region and ethnic groups, and the sample size is limited. The results cannot fully represent the overall population.

\section{Conclusion}

This study further confirms the efficacy and safety of apatinib in the treatment of advanced HCC. The occurrence of apatinib-related hypertension is a potential predictor of a significant prolongation of survival in patients with advanced HCC.

\section{Ethics Approval}

The study protocol was in accordance with the ethical guidelines of the 1975 Declaration of Helsinki and was approved by the Medical Ethics Committee of the Tianjin Medical University Cancer Institute and Hospital (reference number bc2019090).

\section{Informed Consent}

As this was a retrospective review of the medical records and all data have been anonymously processed, obtaining informed consent from the included individuals was not deemed necessary and not requested by the ethical review board.

\section{Disclosure}

This study was supported by the National Science and Technology Major Project (No.2017ZX10203207). The authors declare no conflicts of interest.

\section{References}

1. Bray F, Ferlay J, Soerjomataram I, Siegel RL, Torre LA, Jemal A. Global cancer statistics 2018: GLOBOCAN estimates of incidence and mortality worldwide for 36 cancers in 185 countries. CA Cancer J Clin. 2018;68(6):394-424. doi:10.3322/caac.21492
2. Heimbach JK, Kulik LM, Finn RS, et al. AASLD guidelines for the treatment of hepatocellular carcinoma. Hepatology. 2018;67 (1):358-380. doi:10.1002/hep.29086

3. Llovet JM, Ricci S, Mazzaferro V, et al. Sorafenib in advanced hepatocellular carcinoma. $N$ Engl J Med. 2008;359(4):378-390. doi:10.1056/NEJMoa0708857

4. Cheng A-L, Kang Y-K, Chen Z, et al. Efficacy and safety of sorafenib in patients in the Asia-Pacific region with advanced hepatocellular carcinoma: a phase III randomised, double-blind, placebo-controlled trial. Lancet Oncol. 2009;10(1):25-34. doi:10.1016/S1470-2045(08)70285-7

5. Abou-Alfa GK, Meyer T, Cheng AL, et al. Cabozantinib in patients with advanced and progressing hepatocellular carcinoma. $N$ Engl J Med. 2018;379(1):54-63. doi:10.1056/NEJMoa1717002

6. Kudo M, Finn RS, Qin S, et al. Lenvatinib versus sorafenib in first-line treatment of patients with unresectable hepatocellular carcinoma: a randomised Phase 3 non-inferiority trial. Lancet. 2018;391 (10126):1163-1173. doi:10.1016/S0140-6736(18)30207-1

7. Bruix J, Qin S, Merle P, et al. Regorafenib for patients with hepatocellular carcinoma who progressed on sorafenib treatment (RESORCE): a randomised, double-blind, placebo-controlled, phase 3 trial. Lancet. 2017;389(10064):56-66. doi:10.1016/S0140-6736(16)32453-9

8. Bautch VL. VEGF-directed blood vessel patterning: from cells to organism. Cold Spring Harb Perspect Med. 2012;2(9):a006452. doi:10.1101/cshperspect.a006452

9. Lee S, Chen TT, Barber CL, et al. Autocrine VEGF signaling is required for vascular homeostasis. Cell. 2007;130(4):691-703. doi:10.1016/j.cell.2007.06.054

10. Koch S, Tugues S, Li X, Gualandi L, Claesson-Welsh L. Signal transduction by vascular endothelial growth factor receptors. Biochem J. 2011;437(2):169-183. doi:10.1042/BJ20110301

11. Peng $\mathrm{S}$, Zhang Y, Peng H, et al. Intracellular autocrine VEGF signaling promotes EBDC cell proliferation, which can be inhibited by Apatinib. Cancer Lett. 2016;373(2):193-202. doi:10.1016/j.canlet.2016.01.015

12. Qin SK, Ouyang XN, Bai YX, et al. Multicenter phase II study of apatinib, a novel inhibitor of VEGFR, in patients with advanced hepatocellular carcinoma. ASCO annual meeting. 2014: Abstract 4019.

13. Kong Y, Sun L, Hou Z, et al. Apatinib is effective for treatment of advanced hepatocellular carcinoma. Oncotarget. 2017;8 (62):105596-105605. doi:10.18632/oncotarget.22337

14. Simons M, Eichmann A. "On-target" cardiac effects of anticancer drugs: lessons from new biology. J Am Coll Cardiol. 2012;60 (7):626-627. doi:10.1016/j.jacc.2012.01.069

15. Shah DR, Shah RR, Morganroth J. Tyrosine kinase inhibitors: their on-target toxicities as potential indicators of efficacy. Drug Saf. 2013;36(6):413-426. doi:10.1007/s40264-013-0050-x

16. Nazer B, Humphreys BD, Moslehi J. Effects of novel angiogenesis inhibitors for the treatment of cancer on the cardiovascular system: focus on hypertension. Circulation. 2011;124(15):1687-1691. doi:10.1161/CIRCULATIONAHA.110.992230

17. Hamnvik OP, Choueiri TK, Turchin A, et al. Clinical risk factors for the development of hypertension in patients treated with inhibitors of the VEGF signaling pathway. Cancer. 2015;121(2):311-319. doi:10.1002/cncr.28972

18. Dienstmann R, Brana I, Rodon J, Tabernero J. Toxicity as a biomarker of efficacy of molecular targeted therapies: focus on EGFR and VEGF inhibiting anticancer drugs. Oncologist. 2011;16 (12):1729-1740. doi:10.1634/theoncologist.2011-0163

19. Keizer RJ, Gupta A, Shumaker R, Beijnen JH, Schellens JH, Huitema AD. Model-based treatment optimization of a novel VEGFR inhibitor. $B r J$ Clin Pharmacol. 2012;74(2):315-326. doi:10.1111/j.1365-2125.2012.04197.x

20. Liu X, Qin S, Wang Z, et al. Early presence of anti-angiogenesisrelated adverse events as a potential biomarker of antitumor efficacy in metastatic gastric cancer patients treated with apatinib: a cohort study. J Hematol Oncol. 2017;10(1):153. doi:10.1186/s13045-0170521-0 
21. Fang SC, Huang W, Zhang YM, Zhang HT, Xie WP. Hypertension as a predictive biomarker in patients with advanced non-small-cell lung cancer treated with apatinib. Onco Targets Ther. 2019;12:985-992. doi:10.2147/OTT.S189984

22. Gampenrieder SP, Romeder F, Muss C, et al. Hypertension as a predictive marker for bevacizumab in metastatic breast cancer: results from a retrospective matched-pair analysis. Anticancer Res. 2014;34(1):227-233.
23. Rautiola J, Donskov F, Peltola K, Joensuu H, Bono P. Sunitinibinduced hypertension, neutropaenia and thrombocytopaenia as predictors of good prognosis in patients with metastatic renal cell carcinoma. BJU Int. 2016;117(1):110-117. doi:10.1111/bju.12940

24. Zhong J, Ali AN, Voloschin AD, et al. Bevacizumab-induced hypertension is a predictive marker for improved outcomes in patients with recurrent glioblastoma treated with bevacizumab. Cancer. 2015;121 (9):1456-1462. doi:10.1002/cncr.29234

\section{Publish your work in this journal}

Cancer Management and Research is an international, peer-reviewed open access journal focusing on cancer research and the optimal use of preventative and integrated treatment interventions to achieve improved outcomes, enhanced survival and quality of life for the cancer patient
The manuscript management system is completely online and includes a very quick and fair peer-review system, which is all easy to use. Visit http://www.dovepress.com/testimonials.php to read real quotes from published authors. 